\title{
Helicobacter pylori infection in patients with chronic calcular cholecystitis a cross- sectional study
}

\author{
Amir A. Fikry', Arafat A. Kassem², Doaa Shahin ${ }^{3}$, Hosam Aldeen Salah Shabana ${ }^{2}$, \\ Sadek Mostafa ${ }^{2}$ \\ ${ }^{1}$ Department of Surgery, Faculty of Medicine, Mansoura University ,Mansoura, Egypt \\ ${ }^{2}$ Department of Internal Medicine, Faculty of Medicine, Al-Azhar University ,Cairo, Egypt \\ ${ }^{3}$ Department of Clinical Pathology, Faculty of Medicine, Mansoura University ,Cairo, Egypt
}

\section{Email address:}

amrikry@yahoo.com (A. A. Fikry )

\section{To cite this article:}

Amir A. Fikry, Arafat A. Kassem, Doaa Shahin, Hosam Aldeen Salah Shabana, Sadek Mostafa. Helicobacter Pylori Infection in Patients with Chronic Calcular Cholecystitis a Cross- Sectional Study. Journal of Surgery. Vol. 2, No. 4, 2014, pp. 58-62.

doi: $10.11648 /$ j.js.20140204.12

\begin{abstract}
Aims: Helicobacter pylori was found in the bile and gallbladder, so we are aiming to examine the relationship between H. pylori-like bacteria and chronic calcular cholecystitis. METHODS: This cross-sectional study was conducted at our hospitals from May 2011 to January 2012. One hundred and forty patients with chronic calcular cholecystitis were enrolled in this study. Gastroscopy with gastric or duodenal H.pylori urease test and detection of H. pylori in serum were done before the surgical procedure for all patients. After cholecystectomy, the gallbladder tissue was investigated for H. pylori by rapid urease test and Geimsa stain. RESULTS: Of all 140 patients $62.1 \%$ were females, mean age of our patients was 39.04 \pm 7.3 years. H. pylori IgG antibody titre was positive in 32 patients with biliary duodenitis, in 43 patients with biliary gastritis, and in 18 patients with duodenal ulcer. The gastric rapid urease test was positive in $40.7 \%$. The gallbladder rapid urease test was positive in $25 \%$. In 24 patients both gastric and gallbladder mucosa tested positive for rapid urease test. In 28 patients, the gastric urease test was positive while the gallbladder mucosa tested negative. In 13 patients, the gallbladder mucosa tested positive while the gastric mucosa was negative. There was a significant correlation between the rapid urease test in the gallbladder and in the gastric or duodenal mucosa $(\mathrm{P}=0.002)$. Modified Geimsa staining of gallbladder specimens revealed that $\mathrm{H}$. pylori-like bacteria were detected in $63.6 \%$. There was a significant correlation between histopathological examination of gallbladder mucosa stained with Geimsa stain and the gallbladder rapid urease test $(\mathrm{P}<$ $0.001)$, but not correlated with the gastric urease test $(\mathrm{P}=0.210)$. CONCLUSION: Our study demonstrates that $\mathrm{H}$. pylori is present in the gallbladder mucosa in patients with chronic calcular cholecystitis and might be an etiological cause of chronic inflammation'.
\end{abstract}

Keywords: Cholecystitis, Helicobacter Pylori, Urease Test

\section{Introduction}

Helicobacter pylori is a gram negative bacteria which causes chronic active gastric inflammation, peptic ulceration, gastric carcinoma, and malignant lymphoma of gastric mucosa-related lymphoid tissue (MALToma) $(1,2,3)$. Recently, it has been found that H. pylori have certain relationship to some diseases in the organs besides the stomach and duodenum ( 4 ). Recent work demonstrated the presence of H.pylori and $\mathrm{H}$. bilis both in the bile and gallbladder of more than $75 \%$ of patients with chronic cholecystitis undergoing surgery. It was demonstrated that some species such as entero-hepatic helicobacter could cause chronic active hepatitis in animals and that $\mathrm{H}$. hepaticus was related to the appearance of tumors in the liver of mice (5).

H. pylori organism was detected in bile samples collected by percutaneous transhepatic cholangio-drainage from patients with pancreatic head tumors, suggesting that $\mathrm{H}$. pylori may be associated with asymptomatic cholangitis (6).

The prevalence of infection in the digestive tract by Helicobacter species varies in the population studied, suggesting epidemiological differences in the distribution of 
the bacillus in various countries. The true prevalence and relationship of different species of helicobacter in the pathogenesis of these diseases is not known $(7,8)$.

Cholecystitis is defined as inflammation of the gallbladder that occurs most commonly because of an obstruction of the cystic duct from cholelithiasis. Ninety percent of cases involve stones in the cystic duct (i.e. calculous cholecystitis) with the other $10 \%$ of cases representing acalculous cholecystitis (9). The blocking of cystic duct leads to thickening of bile, bile stasis, and secondary infection by gut organisms predominantly E. coli and bacteroids species (10). As the gallbladder becomes distended, blood flow and lymph drainage are compromised leading to mucosal ischemia and necrosis. The role of Helicobacter pylori in the pathogenesis of gallbladder diseases in humans or even its presence in the gallbladder tissue therefore remains unclear but many researchers have demonstrated the presence of Helicobacter in gallbladder of patients with calcular cholecystitis $(11,12$, and 4).

\section{Patients and Methods}

This cross-sectional study was conducted at our hospitals from May 2011 to March 2012. One hundred and forty patients were included in this study and all presented with symptomatic calcular cholecystitis. Full history of all patients was taken and complete physical and relevant clinical examinations were performed. Routine investigations including liver function tests and total leucocytic count were also sorted. The calcular cholecystitis was diagnosed by abdominal ultrasound performed by expert radiologist. All patients went for cholecystectomy whether by laparoscopy or by open surgery . Gastroscopy with gastric or duodenal H.pylori urease test (Pronto Dry) was done before the surgical procedure for all patients. Patients with acute cholecystitis, cholangitis, biliary and hepatic tumors, crohn's disease, and previous gastric surgery were not considered suitable for evaluation. Patients undergoing endoscopic retrograde cholangiopancreatography (ERCP) and patients who had received $H$. pylori eradication treatment in the last 6 months were also excluded from the study. All patients signed a free and informed consent.

Determination of H. pylori status: After laparoscopic or open cholecystectomy, the gallbladder was opened in the operation room and two strips of tissue were obtained from the infundibulum of the gallbladder. One sample was used for the rapid urease test (Pronto Dry), and the second sample for histopathological examination. For detection of $\mathrm{H}$. pylori in serum, $2-3 \mathrm{ml}$ venous blood sample was collected in a disposable syringe from every relevant patient a day before laparoscopic or open cholecystectomies and send to laboratory for analysis. Paraffin specimens of cholecystitis were sliced into $4 \mu \mathrm{m}$ sections, stained with modified Geimsa stain and sealed with D.P.X for an optic microscopic observation for the existence of $\mathrm{H}$. pylori-like bacteria . Statistical issues was conducted using SPSS 16 .

\section{Results}

One hundred and forty patients with symptomatic calcular cholecystitis undergoing either open or laparoscopic cholecystectomy were enrolled for the study. Out of 140, $87(62.1 \%)$ were females and 53 (37.9\%) were males. The mean age for female patients was $39.9 \pm$ 8.1 (range from $26-55$ ) and the mean age for male patients was $37.7 \pm 5.8$ years (range from $28-45$ ). The mean age of overall study population was $39.04 \pm 7.3$ years. Gender distribution of $\mathrm{H}$. pylori infection in patients with chronic cholecystitis is shown in table 1 .

Table 1. Gender distribution of H. pylori infection in patients with chronic cholecystitis.

\begin{tabular}{lllll}
\hline \multirow{2}{*}{$\begin{array}{l}\text { IgG U/ } \\
\text { mL. }\end{array}$} & Gender & & Total & P value \\
\cline { 2 - 4 } & Male & Female & & \\
\hline$<20$ & $5(9.4 \%)$ & $18(20.7 \%)$ & $23(16.4 \%)$ & \\
$20-49$ & $9(17 \%)$ & $9(10.3 \%)$ & $18(12.9 \%)$ & \\
$50-99$ & $15(28.3 \%)$ & $14(16.1 \%)$ & $29(20.7 \%)$ & $<0.001$ \\
$>100$ & $24(45.3 \%)$ & $46(52.9 \%)$ & $70(50 \%)$ & \\
Total & $53(100 \%)$ & $87(100 \%)$ & $140(100 \%)$ & \\
\hline
\end{tabular}

All patients had agreed to undergo gastroscopy before surgery. The results of gastroscopy revealed: 35 patients (25\%) had biliary duodenitis, 46 patients (32.9\%) had biliary gastritis, 18 patients $(12.9 \%)$ had duodenal ulcers, and 41 patients $(29.3 \%)$ had normal gastroscopy.

H. pylori IgG antibody titre was positive in 32 patients with biliary duodenitis, was positive in 43 patients with biliary gastritis, was positive in 18 patients with duodenal ulcer, but was negative in 3 patients with biliary duodenitis, in 3 patients with biliary gastritis, and also in 41 patients with normal gastroscopy. The IgG antibody titre of $\mathrm{H}$. pylori in patients with chronic cholecystitis patients in relation to gender distribution is shown in table 2. There was a significant correlation between $H$. pylori IgG antibody titre and the results of gastroscopy (Spearman's correlation coefficient $\mathrm{rs}=0.796), \mathrm{P}<0.001$. Table 3 .

Table. 2. Gender distribution of H. pylori infected patients with calcular cholecystitis.

\begin{tabular}{llll}
\hline \multirow{2}{*}{ Cholelithiasis } & H. pylori & Total \\
\cline { 2 - 3 } & Positive & Negative & $53(37.9 \%)$ \\
\hline Male & $48(41.1 \%)$ & $5(21.7 \%)$ & $87(62.1 \%)$ \\
Female & $69(58.9 \%)$ & $18(78.3 \%)$ & $140(100 \%)$ \\
Total & $117(100 \%)$ & $23(100 \%)$ & $<0.001$ \\
\hline
\end{tabular}


Table 3. H. pylori antibody titre with gastroscopy results.

\begin{tabular}{lllllll}
\hline H. pylori IgG antibody & Biliary duodenitis & Biliary gastritis & Duodenal ulcer & Normal & Total & Significance \\
\hline $15-20$ & 3 & 6 & 0 & 23 & 32 & \\
$21-50$ & 3 & 6 & 3 & 3 & 15 & \\
$51-100$ & 13 & 11 & 6 & 9 & 39 & $<0.001$ \\
$>100$ & 16 & 23 & 9 & 6 & 54 & \\
Total & 35 & 46 & 18 & 41 & 140 \\
\hline
\end{tabular}

The rapid urease test in the gastric or duodenal mucosa was positive in 57 patients $(40.7 \%$ ), and negative in 83 patients $(59.3 \%)$. The rapid urease test was positive in the gallbladder mucosa of 35 patients $(25 \%)$, and negative in 105 patients $(75 \%)$. In 24 patients, both gastric and gallbladder mucosa tested positive with the rapid urease test. In 28 patients, the gastric urease test was positive while the gallbladder mucosa tested negative. In 13 patients, the gallbladder mucosa tested positive while the gastric mucosa was negative. There was a significant correlation between the rapid urease test in the gallbladder mucosa and in the gastric or duodenal mucosa $(\mathrm{P}=0.002)$. Table 4 .

Table 4. Rapid urease test results in gastric and gallbladder mucosa.

\begin{tabular}{llll}
\hline Rapid urease test & $\begin{array}{l}\text { H. pylori positivity } \\
\text { N ( \%) }\end{array}$ & $\begin{array}{l}\text { H. pylori Negativity } \\
\text { N ( \%) }\end{array}$ & P value \\
\hline Gastric or duodenal mucosa & $57(40.7 \%)$ & $83(59.3 \%)$ & 0.002 \\
Gallbladder mucosa & $35(25 \%)$ & $105(75 \%)$ & \\
\hline
\end{tabular}

On histological examination after modified Geimsa staining, H. pylori- like bacteria were detected in 89 patients $(63.6 \%)$. In patients with positive histopathological examination for $\mathrm{H}$. pylori-like bacteria, 39 patients from 89 had positive gastric urease test and 18 patients from 51 had negative gastric urease test, thus there was no significant correlation between the two tests ( $\mathrm{P}=$ 0.210 ). There was a significant correlation between the histopathological examination of gallbladder mucosa stained with Geimsa stain and the rapid urease test in the gallbladder mucosa in our patients $(\mathrm{P}<0.001)$. The gallbladder tissue stained with modified Geimsa stain was significantly correlated with $\mathrm{H}$. pylori antibody titre, and with the gallbladder rapid urease test $(P<0.001)$, but not correlated with the gastric or duodenal rapid urease test. Tables 5 and 6.

Table 5. Detection of h. pylori with different tests in our patients.

\begin{tabular}{llll}
\hline & $\begin{array}{l}\text { H. pylori positivity } \\
\text { N (\%) }\end{array}$ & $\begin{array}{l}\text { H. pylori Negativity } \\
\text { N }(\%)\end{array}$ & P value \\
\hline Rapid urease test in gallbladder mucosa & $35(25 \%)$ & $105(75 \%)$ & \\
H. pylori IgG antibody titre & $103(73.6 \%)$ & $37(26.4 \%)$ & $<0.001$ \\
Gallbladder Geimsa staining & $89(63.6 \%)$ & $51(36.4 \%)$ & \\
Rapid urease test in gastric mucosa & $57(40.7 \%)$ & $83(59.3 \%)$ & \\
\hline
\end{tabular}

Table 6. Correlation between gallbladder geimsa staining and gastric rapid urease tests

\begin{tabular}{|c|c|c|c|c|c|}
\hline & \multicolumn{2}{|c|}{ Gastric RUT } & \multirow{2}{*}{ Total } & \multirow{2}{*}{$\mathbf{r}$} & \multirow{2}{*}{ Significance } \\
\hline & Positive & Negative & & & \\
\hline Gallbladder Positivity & 39 & 50 & 89 & 0.374 & 0.210 \\
\hline Gallbladder Negativity & 18 & 33 & 51 & & \\
\hline Total & 57 & 83 & 140 & & \\
\hline
\end{tabular}

\section{Discussion}

The gallbladder and stomach are originated from endoblasts and have similar tissue structures, with the mucosa covered with a slime layer $(13,14)$. AB / PAS stain showed that the epithelial cells of the gallbladder mucosa secreted neutral mucus, with gastric metaplasia, in about $31 \%$ of cases with cholecystolithiasis and the epithelial cells of the gallbladder mucosa had gastric metaplasia in cases with cholecystolithiasis $(15,16)$. Thus significance of gastric metaplasia of gallbladder mucosa in choledocholithiasis lies in that the gastric metaplasia provides conditions for $\mathrm{H}$. pylori colonization in the gallbladder (17).

H. pylori has been implicated as a risk factor for different Extraintestinal disorders. These organisms are tolerant to bile, colonize bile canaliculi and the gallbladder and can cause hepatitis and cholecystitis. Chronic cholecystitis is commonly associated with gallstone disease and some studies have shown that cholecystitis and gallstones can cause epithelial hyperplasia of the gallbladder mucosa or cancer and various bacterial genomes have been detected in the gallbladder carcinoma tissue (18). H. pylori can damage 
human gallbladder epithelial cells and could be the key factor that leads to cholecystitis (19). Manog et al. have demonstrated a strong association between Helicobacter and gallbladder disease (20). Most of patients in our study were females with cholelithiasis $(62.1 \%)$; the female population was also predominant in relation to $\mathrm{H}$. pylori infection in patients with calcular cholecystitis (58.9\%) suggesting that the majority of patients with calcular cholecystitis in our study were positive with higher titres of anti-H. pylori antibodies. Our study is consistent with studies done by Apostolov et al. (4) and Abro et al. (21). Some studies found no significant differences in the prevalence of $\mathrm{H}$. pylori $\mathrm{IgG}$ antibodies in cholelithiasis patients and controls (12, 26, and 27).

By means of gastroscopy, we found a good correlation between the gastroscopy results and gastric rapid urease test $(r=0.452)$, with gallbladder urease test $(r=0.386)$, with gallbladder tissue stained with Geimsa stain $(\mathrm{r}=$ $0.552)$, and with $H$. pylori $\operatorname{IgG}$ antibody titre $(r=0.809$, for all the $\mathrm{P}$ value was $<0.001$. According to the previous results, we found an increasing rate of bile reflux in our patients with chronic calcular cholecystitis. In our study, according to the gallbladder tissue stained with Geimsa staining, there were $35(25 \%)$ with biliary duodenitis, 27 $(19.3 \%)$ with biliary gastritis, 18 patients $(12.9 \%)$ with duodenal ulcer, and 51 patients $(36.4 \%)$ with negative Geimsa staining of gallbladder tissue. According to the gallbladder rapid urease test, there were 11 patients $(7.9 \%)$ with biliary duodenitis, 12 patients $(8.6 \%)$ with biliary gastritis, 11 patients $(7.9 \%)$ with duodenal ulcer, and 105 patients $(75 \%)$ with negative gallbladder rapid urease test. Lastly, according to H. pylori IgG antibody titre, there were 35 patients $(25 \%)$ with biliary duodenitis, 46 patients $(32.9 \%)$ with biliary gastritis, 18 patients $(12.9 \%)$ with duodenal ulcer, and 41 patients $(29.3 \%)$ with normal gastroscopy. These results indicate that patients with chronic cholecystitis are apt to bile regurgitation which occurs more commonly in our patients. These results agree with the findings done by Silva et al., they stated that patients with chronic calcular cholecystitis are apt to bile regurgitation which occurs in $80 \%$ of patients with chronic cholecystitis, while in only $32 \%$ of normal person (11). $\mathrm{H}$. pylori organisms are sensitive to bile salts especially the unconjugated bile salts that exert poisonous effect on $\mathrm{H}$. pylori that can't live in an environment with bile salts. In our patients with chronic cholecystitis whose exposed to bile regurgitation have a high rate of $H$. pylori in their stomachs, suggesting that a kind of $\mathrm{H}$. pylori that can resist bile salts exist and can survive, and, in combination with the bile, aggravate the injury of gastric mucosa (22), thus $\mathrm{H}$. pylori that resist the bile salts and survive under basic conditions can enter the gallbladder via an inverse infection route through the common passage. In a study done by Chen et al., they found that in the stomachs of patients with chronic lithic cholecystitis and a bile reflux, H. pylori were present in up to $46.1 \%$ ( $35 / 76$ ), higher than $44.4 \%$ ( $16 /$ 36 ) in patients without bile reflux. It indicates that a high incidence rate of $H$. pylori infection still existed in the stomach although there was a bile reflux (17). Our results are consistent with that of Abro et al. and Chen et al. (21, 19).

The rapid urease test is easy to use and reliable. This test was positive in 57 patients $(40.7 \%)$ of gastroscopies performed in our study. In 24 , the urease test was positive in both the gastric and gallbladder mucosa. Of 28 patients with positive gastric rapid urease test, the gallbladder urease test was negative and in 13 patients, the gallbladder mucosa tested positive while the gastric mucosa rapid urease test was negative with a positive significant correlation between these two tests $(\mathrm{P}=0.002)$. This indicates that there was a close relationship of the common presence of $\mathrm{H}$. pylori in both organs.

On histological examination after Geimsa staining, $\mathrm{H}$. pylori-like bacteria were found in $63.6 \%$ of 140 gallbladder specimens and this was correlated with a study done by Chen et al (17). In our study, there was no significant correlation between histopathological examination of gallbladder mucosa stained with Geimsa stain and gastric urease test, but there was a significant correlation between gallbladder tissues stained with Geimsa stain with both H. pylori antibody titre and the gallbladder rapid urease test. Previous studies with different methods have revealed the presence of $\mathrm{H}$. pylori in the biliary tract in $50-60 \%$ of patients studied $(23,24)$. H. pylori in the gastric and duodenal mucosa are much more common. In Egypt and other countries, H. pylori is found in about $80 \%$ of the population (25). Our suggestion is that, chronic $\mathrm{H}$. pylori infection in the gallbladder mucosa may impair gallbladder motility, increase precipitation of cholesterol to form stones and may initiate gallstone formation through urease induced calcium precipitation by Helicobacter species. We conclude that $H$. pylori is present in the gallbladder mucosa and may be one of the etiological factors leading to cholecystitis, which requires further verification.

\section{References}

[1] Arnaout AH, Abbas SH, and Shousha S. H. pylori is not identified in areas of gastric metaplasia of gall bladder. J. Pathol., 160: 333-334, 1990.

[2] Monstein HJ, Jonsson Y, Zdolsek J, Svanvik J. Identification of Helicobacter pylori DNA in human cholesterol gallstones. Scand j. Gastroenterol 2002; 37: 112-119.

[3] Farinato F, Cardin R, Russo VM, Busatto G, Franco M, Rugge M. H. pylori Cag A status, mucosal oxidative damage and gastritis phenotype: a potential pathway to cancer? Helicobacter 2003; 8: 227-234.

[4] Apostolov E, Alsoud WA, Nilson I, Kornilovska I, Usenko V, Lyzogubov V. Helicobacter pylori and other Helicobacter species in gallbladder and liver of patients with chronic cholecystitis detected by immunological and molecular methods. Scan J. Gastroenterol 2005; 40(1): 96-102. 
[5] Bohr UR, Kuestewr D, Meyer F. Low prevalence of Helicobacteraceae in gallstone disease and gallbladder carcinoma in the German population. Clin. Microbiol. Infect 13: 525-531, 2007.

[6] Chen DF, Hu L, Yi P, Liu WW, Fang DC, Cao H. Relationship between Helicobacter pylori and chronic cholecystitis. World Chin J. Digestol 2004; 12(8): 18401843.

[7] Goodman KJ, Cockburn M. The role of epidemiology in understanding the health effects of Helicobacter pylori. Epidemiology 2001; 12: 266-271.

[8] Devrajani BR, Shah SZA, Soomro AA, Devrajani T. Type 2 diabetes mellitus: A risk factor for Helicobacter pylori infection: A hospital based case-control study. Int J. Diabetes Dev. Ctries 2010; 30: 22-26.

[9] Huffman JL, Schenker S. Acute acalculous cholecystitis- a review. Clin. Gastroenterol Hepatol. 2010; 8(1): 15-22.

[10] Ballal M, Jyothi KN, Antony B, Arun C, Prabhut T, Shivanada PG. Bacteriological spectrum of cholecystitis and its antibiogram. Indian J. Med Microbiol 2001; 19: 212-214.

[11] Silva CP, Pereira-lima JC, Oliveira AG, Guerra JB, Marques DL, Sarmanho L. Association of the presence of Helicobacter in gallbladder tissue with cholelithiasis and cholecystitis. J. Clen Microbiol 2003; 41: 5615-8.

[12] Mendez SN, Pichardo R, Gonzalez J, Sanchez H, Moreno M, Barquera E. Lack of association between Helicobacter species colonization and gallstone disease. J. Clin Gastroenterol 2001; 32: 138-141.

[13] Randi G, Franceschi S, Vecchina C. Gallbladder cancer worldwide geographical distribution and risk factors. Int J. Cancer 2006; 118: 1591-1602.

[14] Pradhan SB, Dali S. Relation between gallbladder neoplasm and Helicobacter hepaticus infection. Kathmandu Univ. Med J (KUM) 2004; 2: 331-335.

[15] Jiano JZ, Nie QH, Zhao CL, Wu YS, Wen SX, Wu Q. Clinical relationship between Helicobacter pylori infection and chronic hepatopathy. Shijie Huaren Xiaohua Zazhi 2003; 11: 851-853.

[16] Chen W, Li D, Cannon RJ, Stubbs RS. Common presence of Helicobacter DNA in the gallbladder of patients with gallstone diseases and controls. Dig Liver Dis 2003; 35: 237-243.
[17] Chen DF, Hu L, Yi P, Liu WW, Fang DC, Cao H. H.pylori are associated with chronic cholecystitis. World J. Gastroenterol 2007; 13(7): 1119-1122.

[18] Maurer KJ, Carey MC, Fox JG. Role of infection, inflammation, and the immune system in cholesterol gallstone formation. Gastroenterology 2009; 136: 425-40.

[19] Chen DF, Hu L, Yi P, Liu WW, Fang DC, Cao H. Helicobacter pylori damages human gallbladder epithelial cells in vitro. World J. Gastroenterol 2008; 14: 6924-8.

[20] Manoj P. Helicobacter species are associated with possible increase in risk of biliary lithiasis and benign biliary diseases. World J. Surg Oncol 2007; 5: 94-96.

[21] Abro AM, Haider IZ, Ahmad S. Helicobacter pylori infection in patients with calcular cholecystitis. A hospital based study. J. Ayub Med Coll Abbottabad 2011; 23(1): 3033

[22] Bulajic M, Maisonneuve P, Schnieder-Brachert W, Muller P, Reischl U, Stimach B, Lehn N, Lowenfels AB, Lohr M. Helicobacter pylori and the risk of benign and malignant biliary tract disease. Cancer 2002; 95: 1946-1953.

[23] Ghzal A, Elsabbagh N, El-Riwini M. Presence of Helicobacter Spp. DNA in the gallbladder of Egyptian patients with gallstone diseases. EMHJ. 17(12): 925-29.

[24] Jafri D, Singhal S, Malik A, Khan A. Helicobacter pylori in gallbladder disease. Biomedical Research 2010; 21(4): 437 440 .

[25] World Gastroenterology Organization "world gastroenterology organization global guidelines: helicobacter pylori in developing countries". Journal of Digestive Diseases, Vol.12, pp 319-326, 2011.

[26] Myung SJ, Kim MH, Shim KN, Kim YS, Kim HJ. Detection of Helicobacter pylori DNA in human biliary tree and its association with hepolithiasis. Dig Dis Sci 2000; 45: 14051412.

[27] Figura N, Cetta F, Angelico M, Montalto G, Getta D, Pacenti L. Most Helicobacter pylori infected patients have specific antibodies and some also have $\mathrm{H}$. pylori antigen and genomic material in bile: is it a risk factor for gallstone formation? Dig Dis Sci 1998; 43: 854-62. 